\footnotetext{
${ }^{2}$ P. A. Fleury and R. Loudon, Phys. Rev. 166, 514 (1968).

${ }^{3}$ T. Moriya, J. Appl. Phys. 39, 1042 (1968).

${ }^{4}$ R. J. Elliott and M. Thorpe, J. Phys. C: Proc. Phys.

Soc., London 2 , 1630 (1969).

${ }^{5}$ P. A. Fleury, Phys. Rev. 180, 591 (1969).

${ }^{6}$ T. Kawasaki, J. Phys. Soc. Jap. 29, 1144 (1970).

${ }^{7}$ P. S。 Pershan and A. Oseroff, Phys. Rev. B 1,2359 (1970).

${ }^{8}$ S. R. Chinn and H. J. Zeiger, Phys. Rev. Lett. 21, 1589 (1968); P. A. Fleury, J. Appl.Phys. $\underline{41}, 886$ (1970).

${ }^{9}$ C. P. Slichter, Principles of Magnetic Resonance

(Harper and Row, New York, 1963), pp. 226-230.

${ }^{10}$ D. S. Ritchie and M. E. Fisher, Phys. Rev. B (to be published).

${ }^{11}$ Our justifications for applying ferromagnetic cor-
}

relations to antiferromagnetic $\mathrm{MnF}_{2}$ are that, in magnitude, correlations for the ferromagnetic and antiferromagnet are identical in the limit $S \rightarrow \infty$, as pointed out by Ritchie and Fisher; and, as is well known, the thermodynamics of spin- $\frac{5}{2}$ systems $\left(\mathrm{Mn}^{2+}\right)$ are practically the same as for classical $(S \rightarrow \infty)$ systems.

${ }^{12}$ A. Okazaki, K. C. Turberfeld, and R. W. Stevenson, Phys. Lett. $\underline{8}, 9$ (1964).

${ }^{13}$ V. Jaccarino and R. G. Shulman, Phys. Rev. 107, 1196 (1957); P. Heller and G. B Benedek, Phys. Rev. Lett. 8 , 428 (1962); G. G. Low, Proc. Phys. Soc., London 82 , 992 (1963).

${ }^{14} \mathrm{~A}$ complete magnetization curve for $\mathrm{MnF}_{2}$, which combines the results of Ref. 13, may be found in F. Keffer, in Handbuch der Physik, edited by S. Flügge

(Springer, Berlin, 1966), Vol. 18, p. 127.

${ }^{15}$ L. P. Kadanoff et al., Rev. Mod. Phys. $\underline{39}, 395$ (1967).

\title{
Variable Masses in Fission and Heavy-Ion Collisions*
}

\author{
Peter Lichtner and Dieter Drechsel \\ Institut fur Kernphysik der Universität Mainz, Mainz, Germany
}

and

Joachim Maruhn and Walter Greiner

Institut für Theoretische Physik der Universität Frankfurt, Frankfurt am Main, Germany

(Received 20 December 1971)

\begin{abstract}
With the use of the cranking formula, the coordinate-dependent mass parameters of the kinetic-energy operator in fission processes and heavy-ion collisions are calculated in the two-center oscillator model. It is shown that the reduced mass and also the classical moment of inertia are obtained for large separations of the fragments. For small separations, however, the mass parameter for the motion of the centers of mass of the fragments is larger than the reduced mass by an order of magnitude.
\end{abstract}

The double-center shell model developed during the last few years ${ }^{1-3}$ has been successful in describing fission phenomena as well as heavy-ion scattering. However, up to now the variation of the inertial parameters in this model has not been studied. In fact, the discussion of fission in previous work has been confined to the mapping of potential energy surfaces, and the treatment of heavy-ion scattering has been carried out under the assumption of a constant reduced mass.

In this paper we show that the inertial parameters change very rapidly, particularly when the two fragments or ions have a large overlap. Thus the fission lifetimes and the cross sections for heavy-ion, scattering at high energies will be strongly affected.

The effect of a variable mass has been studied by Hofmann and Dietrich ${ }^{4}$ in a one-dimensional model using several phenomenological forms for the mass variation. Similarly Updegraff and Onley ${ }^{5}$ have included this effect in a three-dimensional case in their description of photofission in the dynamic collective model.

Griffin ${ }^{6}$ has stressed the importance of the Landau-Zener ${ }^{7}$ effect of level crossing on the mass parameters and estimated that the masses should be higher than the reduced mass by at least an order of magnitude. A similar conclusion has been reached by Sobiczewski et al..$^{8}$ in the case of $\beta$ vibrations and in recent unpublished work. ${ }^{9}$ The advantage of the double-center shell model used in the present calculations is its ability to describe the complete fission process to the stage of two separated fragments and, furthermore, its applicability to heavy-ion scattering. In this note we restrict ourselves to the symmetric double-center oscillator which is described 
by two variables: the coordinate $R$, which is half the separation of the centers of the two potentials, and $\beta$, which describes the deformation of the two fragments. ${ }^{1,3}$ As illustrated in Fig. 1, $\beta$ is given by the ratio of the two principal axes of the equipotential surface. Thus $\beta$ less than, equal to, or greater than 1 refers to an oblate, spherical, or prolate shape, respectively. In our calculation we used the same potential and parameters as given by Scharnweber, Greiner, and Mosel. ${ }^{3}$ We allow for the rotation of the system by including two angles $\theta$ and $\varphi$, describing the orientation of the axis of symmetry. To second order in velocities, the classical kinetic energy will then have the form

$$
\begin{aligned}
T=\frac{1}{2} B_{R R} \dot{R}^{2}+B_{B R} \dot{R} \dot{\beta}+ & \frac{1}{2} B_{\beta B} \dot{\beta}^{2} \\
& +\frac{1}{2} I\left(\dot{\theta}^{2}+\sin ^{2} \theta \dot{\varphi}^{2}\right),
\end{aligned}
$$

where the inertial parameters are functions of the coordinates $R$ and $\beta$. We calculate the inertial parameters with the cranking model ${ }^{10,11}$ which gives, for two collective coordinates $x$ and $y$, the mass

$$
\begin{aligned}
B_{x y} & =2 \hbar^{2} \sum_{i j} \frac{\langle 0|(\partial / \partial x)| i j\rangle\langle i j|(\partial / \partial y)| 0\rangle}{E_{i}+E_{j}} \\
& =2 \hbar^{2} \sum_{i j} \frac{\langle 0|(\partial H / \partial x)| i j\rangle\langle i j|(\partial H / \partial y)| 0\rangle}{\left(E_{i}+E_{j}\right)^{3}},
\end{aligned}
$$

and similarly for the moment of inertia, $I$. Here $|0\rangle$ denotes the BCS ground state, $|i j\rangle$ is a twoquasiparticle state, and $E_{i}$ and $E_{j}$ are the quasiparticle energies.

The two collective coordinates $R$ and $\beta$, although they describe somewhat restricted shapes, should give the main features of fission. The initial stage of fission is expected to be mainly a $\beta$ deformation, while the coordinate $R$ allows one to describe the fission process even up to the final stage of separation of the two fragments. At

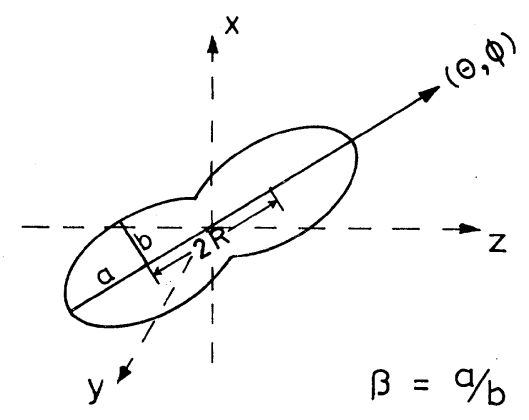

FIG. 1. Shape of the potential in the two-center shell model. large deformations these two coordinates clearly describe different shapes. However, even at small deformations these coordinates are never equivalent in that the shape given by small values of $R$ contains all even multipoles (i.e., to first order in $R$ all even multipole moments are present), while in the limit of small $\beta$ only the quadrupole moment is nonzero. We note that a removal of our restriction to cylindrically symmetric shapes will modify Eq. (1) by introducing three different moments of inertia. In high-energy heavy-ion scattering the restriction to quadratic terms in the velocity may not be very good, and fourth- and higher-order terms need to be studied. They correspond to a velocity dependence of the variable mass.

Figure 2 shows the calculated mass parameters plotted against $R$ for oblate and prolate shapes for ${ }_{92}^{236} \mathrm{U}$. As expected, the mass $B_{R R}$ approaches the reduced mass for large separation [two curves in Fig. 2(a)]. One can in fact easily show analytically that the double-center oscillator used here produces asymptotically the correct reduced mass. Below the scission point, however, the effective mass becomes considerably larger than the reduced mass and shows strong fluctuations due to the variation of the ground-state pairing structure, i.e., the variation of the BCS occupation probabilities $V_{i}$. This was first pointed out by Belyaev. ${ }^{10}$ Beyond the scission point, however, the occupation probabilities become constant, and the mass is solely determined by the variation of the single-particle wave functions with deformation. The latter contribution gives rise to a smooth background and approaches the reduced mass.

The mass parameter $B_{R R}$ is the mass in terms of the coordinates $R$ and $\beta$ of the double-center shell model, and should be used in connection with the potential-energy surfaces $V(R, \beta)$ calculated in that model. It should be kept in mind, however, that the coordinate $R$ does not coincide with the distance $\rho$ between the centers of mass of the two fragments. In fact, this distance increases much slower than $R$ below the scission point and only asymptotically do the two distances coincide. In general

$$
B_{\rho \rho}=B_{R R}(d R / d \rho)^{2},
$$

where

$$
\rho=A^{-1} \sum_{i}\langle i\|z\| i\rangle V_{i}^{2},
$$

with the BCS occupation probabilities $V_{i}{ }^{2}$. The resulting mass $B_{\rho \rho}$ is considerably larger than 

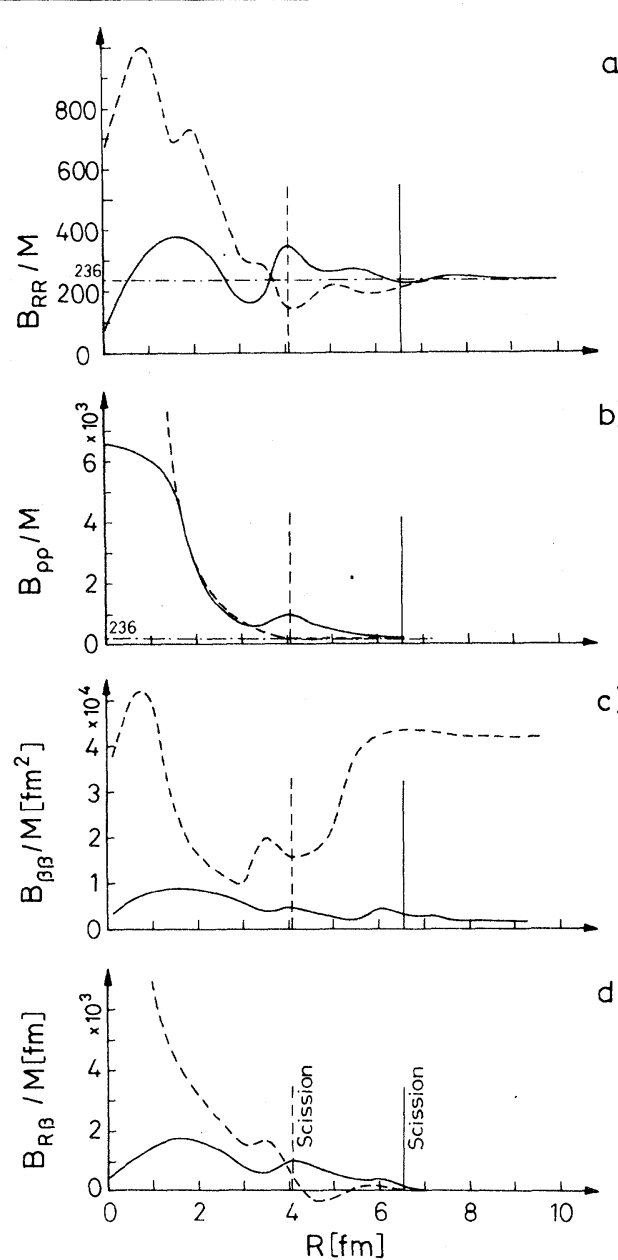

FIG. 2. Mass parameters in units of nucleon mass $M$ as functions of the separation $R$ of the fragments for $\beta=0.6$ (dashed line) and $\beta=1.2$ (solid line). The vertical lines give the scission points $R_{\mathrm{sc}}$ for the two values of $\beta ; R_{\mathrm{sc}}=1.2(A / 2)^{1 / 3} \beta^{2 / 3}$. The masses $B_{\rho \rho}$ shown in (b) are related to $B_{R R}$ by Eq. (3). (Note that all masses are finite at the origin.)

$B_{R R}$ as may be seen in Fig. 2(b). This demonstrates the striking coordinate dependence of the mass parameters and shows that great care must be taken in comparing masses calculated in different coordinates (models). $B_{\rho \rho}$ is in good agreement with the experimental value given by Bjørnholm. ${ }^{9}$

The mass parameters $B_{B \beta}$ are given in Fig. 2(c). They show fluctuations about a value higher than the irrotational value by a factor of about $5-10$. For large $R$ the mass $B_{\beta \beta}$ reaches a constant value, twice the mass parameter for $\beta$ vibrations in the individual fragments, because the two-center model describes simultaneous $\beta$ vibrations in both fragments. The interference term $B_{B R}$
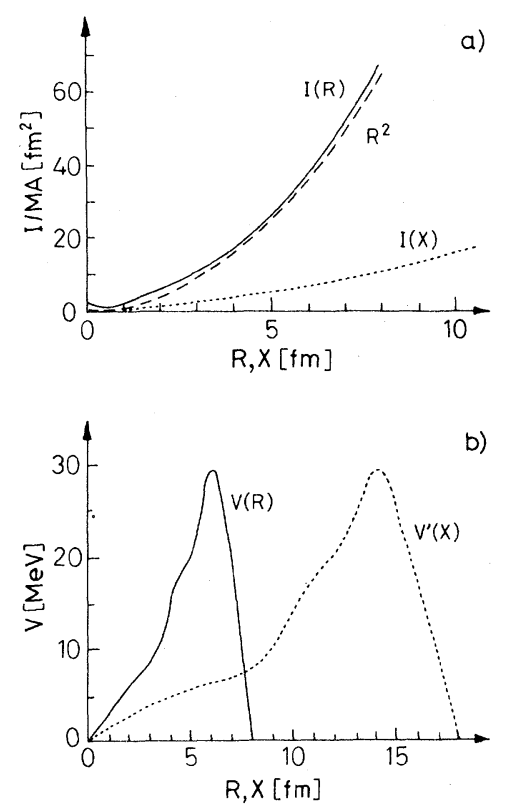

FIG. 3. Illustration of the coordinate transformation Eq. (5) on the moment of inertia, $I$, and the potential $V$, as a function of the separation $R$ and the stretched coordinate $X$ along the path $\beta=0.6 . \quad V^{\prime}$ is defined in Eq. (6a).

shown in Fig. 2(d) approaches zero past the scission point, where the motion in $R$ and $\beta$ becomes decoupled. Finally, the moment of inertia, $I$, given in Fig. 3(a) may be written as the sum of two terms, one describing the rotation of the two potential centers and the other describing the rotation of the two fragments about their centers. For small values of $R$, the moment of inertia approaches that of the latter term. For large separations the first term dominates and approaches the value $M A R^{2}$ [Fig. 3(a)] with $M$ the mass of a nucleon and $A$ the mass-number insert.

For applications to fission or heavy-ion collision, the four-dimensional problem of Eq. (1) may be reduced to three dimensions by choosing one particular fission (reaction) path $\beta=\beta(R)$. Using the prescription of Pauli and Podolsky ${ }^{12}$ to quantize Eq. (1), we obtain the Schrödinger equation

$$
\left(-\frac{\hbar^{2}}{2 \sqrt{B} I} \frac{\partial}{\partial R} \frac{I}{\sqrt{B}} \frac{\partial}{\partial R}+\frac{L^{2}}{2 I}+V\right) \psi=E \psi,
$$

where $B=B_{R R}+(d \beta / d R) R_{R \beta}+\left(d^{2} \beta / d R^{2}\right) B_{B \beta}$. This expression may be simplified by techniques which are a combination of the methods of Refs. 4 and 5 . We replace the wave function $\psi$ by $(M A / I)^{1 / 2} \Phi$ and change to a new coordinate basis by the transformation

$$
X(R)=\int_{0}^{K}\left[B\left(R^{\prime}\right) / M A\right]^{1 / 2} d R^{\prime} .
$$


Equation (4) then becomes

$$
\left(-\frac{\hbar^{2}}{2 M A} \frac{\partial^{2}}{\partial X^{2}}+\frac{L^{2}}{2 I(X)}+V^{\prime}(X)\right) \Phi=E \Phi,
$$

where

$$
V^{\prime}(X)=V(X)+\frac{\hbar^{2}}{2 M A} \frac{d^{2}[I(x)]^{1 / 2} / d X^{2}}{[I(X)]^{1 / 2}}
$$

and the volume element is given by

$$
|\psi|^{2} d v=|\psi|^{2}\left(\frac{B}{M A}\right)^{1 / 2} \frac{I}{M A} d R d \Omega=|\Phi|^{2} d X d \Omega .
$$

Equation (6) is the Schrödinger equation for a system with mass $M A$, constant except for the variable moment of inertia and the additional potential produced by the quantization procedure. Further, because of the simple form of the volume element, the effects of the variable inertia parameters are now completely contained in the potential energy. In order to illustrate this, Fig. 3 shows the effect of the above transformation for the path $\beta=0.6$ (not the fission path!). The additional potential in Eq. (6) does not change the potential appreciably. The main effect of the stretching transformation (5) is an increase of the width of the potential barrier. Calculations are in progress to obtain mass parameters not only in the $\beta-R$ plane but also to include the asymmetric case.

We would like to thank Professor J. J. Griffin, Professor R. Nix, and Professor W. Scheid for several clarifying discussions. We are also grateful to W. Fabian for help in the calculations and particularly to Dr. R. Fraser for his initial collaboration.

*Work supported by the Bundesministerium für Bildung und Wissenschaft, by the Deutsch Forschungsgemeinschaft, and by GSI.

${ }^{1}$ P. Holzer, U. Mosel, and W. Greiner, Nucl. Phys. A138, 241 (1969).

${ }^{2} \mathrm{U}$. Mosel, D. Scharnweber, and W. Greiner, Phys. Rev. Lett. 24, 601 (1970); K. Albrecht, D. Scharnweber, W. Greiner, and U. Mosel, Phys. Lett. 32B, 229 (1970).

${ }^{3}$ D. Scharnweber, W. Greiner, and U. Mosel, Nucl. Phys. A164, 257 (1971); U. Mosel and H. W. Schmitt, Nucl. Phys. A165, 73 (1971), and "Fragment Shell Influences in Nuclear Fission" (to be published).

${ }^{4} \mathrm{D}$. Hofmann and K. Dietrich, to be published.

${ }^{5}$ W. E. Updegraff and D. S. Onley, Nucl. Phys. A161, 191 (1971).

${ }^{6}$ J. J. Griffin, Nucl. Phys. A170, 395 (1971).

${ }^{7}$ C. Zener, Proc. Roy. Soc., Ser. A 137, 696 (1932).

${ }^{8}$ A. Sobiczewski et al., Nucl. Phys. $\overline{\mathrm{A} 131}, 67$ (1969).

${ }^{9} \mathrm{~S}$. Bjфrnholm, in Proceedings of the Nordic-Dutch Accelerator Symposium, Ebeltoft, May 1971 (unpublished); M. Brack, F. Darmgaard, H. C. Pauli, A. Stenholm-Jensin, V. M. Strutinsky, and C. Y. Wong, to be published.

${ }^{10}$ S. T. Belyaev, Kg1. Dansk. Vidensk. Selsk., Mat.Fys. Medd. 31, No. 11 (1959).

${ }^{11}$ D. R. Bés, Kgl. Dansk. Vidensk. Selsk., Mat.-Fys. Medd. 33 , No. 2 (1961).

${ }^{12}$ W. Pauli, in Handbuch der Physik, edited by H. Geiger and K. Scheel (Springer, Berlin, 1933), Vol. 24, Part 1, p. 120; B. Podolsky, Phys. Rev. 32, 812 (1928); see also J. M. Eisenberg and W. Greiner, Excitation Mechanisms of the Nucleus (North-Holland, Amsterdam, 1970), Vol. 2, Chaps. 6,2.

\title{
Intrashell Quartet Excitations in sd-Shell Nuclei
}

\author{
L. Satpathy, K. W. Schmid, and Amand Faessler \\ Institut für Kernphysik der Kernforschungsanlage Jülich, Jülich, West Germany
}

(Received 18 October 1971)

\begin{abstract}
In addition to the quartet excitations between major oscillator shells (intershell quartet states), we propose quartet excitations within a shell (intrashell quartet states). A phenomenological quartet shell model is devised to predict the energies of these states. Evidence for such excitations is also found in a microscopic multiconfiguration Hartree-Fock calculation.
\end{abstract}

Within the last year theoretical ${ }^{1-3}$ and experimental ${ }^{4-8}$ evidence has been accumulating for the existence of weakly interacting quartets consisting of four strongly correlated particles, two protons and two neutrons, in nuclei. The quartet states predicted by Arima, Gillet, and Ginocchio ${ }^{1}$ are assumed to result from the excitation of four correlated particles from one major oscillator shell to another (hereafter referred to as intershell quartet states). We feel that in addition to the existence of intershell quartet states there can be low-lying states originating from the excitation of quartets in the same major shell (hereafter referred to as intrashell quartet states). 